\title{
Significant Spike-Specific IgG and Neutralizing Antibodies in Mice Induced by a Novel Chimeric Virus-Like Particle Vaccine Candidate for Middle East Respiratory Syndrome Coronavirus
}

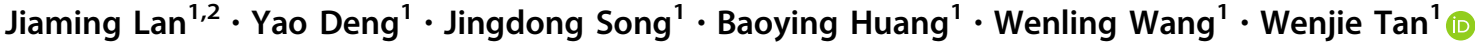

Received: 28 August 2018/Accepted: 11 October 2018/Published online: 29 October 2018

(C) Wuhan Institute of Virology, CAS and Springer Nature Singapore Pte Ltd. 2018

Dear Editor,

Middle East respiratory syndrome coronavirus (MERS$\mathrm{CoV}$ ), first isolated in 2012, has emerged zoonotically among humans (van Boheemen et al. 2012). Since then, MERS-CoV continues to be a public health concern, with a fatality rate of $35 \%$. On-going MERS-CoV outbreaks highlight the urgent need for the development of interventional measures, including an effective vaccine against MERS-CoV infection. Currently, no licensed therapeutic or vaccine is available (Okba et al. 2017).

MERS-CoV spike (S) protein mediates coronaviral penetration into host cells via initial interaction with the host cell surface receptor. Based on the major immunodominant region of $\mathrm{S}$ protein, vaccines against MERS$\mathrm{CoV}$ infection have been developed using purified coronaviral $\mathrm{S}$ protein and DNA or viral vector-based vaccines expressing full-length MERS-CoV S protein or part of the $S$ protein (Okba et al. 2017). However, efforts are required to achieve optimal protective responses, such as a higher titer of neutralizing antibody and protection against MERS$\mathrm{CoV}$ infection.

Virus-like particles (VLPs) are similar in size and morphology to intact virions, thereby rendering them ideal candidates as antigens or immunogens. Since VLPs cannot replicate in humans or revert to a pathogenic form, they are suitable candidates for vaccine development, especially those against life-threatening viruses such as SARS-CoV or MERS-CoV, which cause high morbidity and death. By co-

Wenjie Tan

tanwj28@163.com

1 MOH Key Laboratory of Medical Virology, National Institute for Viral Disease Control and Prevention, Chinese Center for Disease Control and Prevention, Beijing 102206, China

2 Department of Pathogenic Biology, Hebei Medical University, Shijiazhuang 050017, China expressing the $S$, envelope $(E)$, and membrane $(M)$ genes, Wang et al. generated VLPs of MERS-S (2017). However, it is difficult to generate these VLPs in large amounts owing to the lack of an efficient viral core protein. Chimeric VLPs (cVLPs) of SARS-CoV, containing the SARS $S$ protein and the matrix protein of influenza virus using the baculovirus insect cell expression system, completely protected mice from death when administered intramuscularly or intranasally in the absence of an adjuvant (Liu et al. 2011). The use of influenza M1 as a core protein to generate cVLPs for other viruses is reportedly a promising approach, potentially increasing the yield and activating antigen-specific B cells for differentiation and antibody secretion (Liu et al. 2011). In the present study, we developed a novel type of cVLPs containing modified MERS-CoV $S$ protein and the avian influenza matrix 1 (M1) protein, using a baculovirus insect cell expression system.

We first constructed a recombinant plasmid pFastBacDual-M1-St/HAk, using a similar strategy to that described previously (Liu et al. 2011), wherein a modified spike (St/HAk) of MERS-CoV (aa 1 to 1295 of MERS-CoV Spike, codon optimized DNA sequence based on Genbank:DQ420166.1, fused to aa 531-568 of H5N1 HA) and matrix protein 1 (M1) of A/Anhui/01/2005 (H5N1) (aa 1 to 758 of H5N1 M1 codon optimized DNA sequence based on GenBank: HM172159.1) were expressed, as shown in Fig. 1A. To construct the St/HAk, the transmembrane and carboxyl termini (TM/CT, aa 1296 to 1353 of MERS-CoV Spike) of MERS S protein were replaced with the corresponding segment (TM/CT) of HA-derived A/Anhui/01/ 2005 (H5N1). Thus, the recombinant St/HAk gene contained the ectodomain of MERS-CoV Spike and TM/CT of H5N1 HA. pFastBacDual-M1-St/HAk was digested with restriction enzymes $K p n I$ and NheI. Further sequenced to ensure the accuracy of the sequences of M1 and St/HAk. The tandem chimeric S/M1 plasmid pFastBacDual-M1-St/ HAk was transformed into competent cells of DH10Bac ${ }^{\mathrm{TM}}$ 
(Invitrogen, California, USA) to generate recombinant bacmids (Bacmid M1-St/HAk), and verified via PCR. Purified recombinant bacmid DNA was transfected into Sf9 cells (ATCC CRL-1711) to generate recombinant baculoviral M1-St/HAk, using a Bac-to-Bac baculoviral expression system (Invitrogen). Harvested Sf9 cells infected with baculovirus M1-St/HAk were lysed with SDSPAGE buffer. Recombinant proteins were identified via Western blot analysis and indirect immunofluorescence (Lan et al. 2014; Deng et al. 2018).

Thereafter, we harvested the P3 stock of recombinant baculovirus M1-St/HAk from infected Sf9 cells at a titer of $8 \times 10^{7} \mathrm{PFU} / \mathrm{mL}$. Suspended High Five ${ }^{\mathrm{TM}}$ cells were infected by recombinant baculovirus M1-St/HAk at a multiplicity of infection (MOI) of 1,5 , and 10, and harvested at different time points $(24,72$, and $96 \mathrm{~h})$. Protein expression was analyzed via SDS-PAGE and Western blot analysis to determine the optimal conditions. Recombinant
M1-St/HAk protein expression was highest at a MOI of 5 after $72 \mathrm{~h}$. After large-scale amplification, the recombinant proteins yielded bands at approximately $\sim 157 \mathrm{kDa}$, which represents a monomer of the $\mathrm{S}$ protein ectodomain, $\mathrm{a} \sim 110-\mathrm{kDa}$ band, which represents the cleavage of $\mathrm{S}$ glycoprotein into an amino-terminal domain (S1), and a $25-\mathrm{kDa}$ band, which represents the M1 protein of $\mathrm{H} 5 \mathrm{~N} 1$ (Fig. 1B), thereby confirming MERS-CoV $S$ protein and H5N1 M1 protein expression. After purification via ultracentrifugation at $4{ }^{\circ} \mathrm{C}, 3,000 \mathrm{rpm}$ for $2 \mathrm{~h}$, the cVLPs of MERS-S were negatively stained and observed via transmission electron microscope. Enveloped VLPs displayed a spheroid morphology, with a diameter of 80-100 nm and displayed morphological similarity with $\mathrm{CoV}$ virions, with enveloped spikes arranged in a crown shape (Fig. 1C-a). After labelling with murine monoclonal antibodies against $\mathrm{S}$ protein of MERS-CoV and gold-labeled goat anti-mouse IgG antibody, $\mathrm{S}$ specific proteins shown as black pellets
A

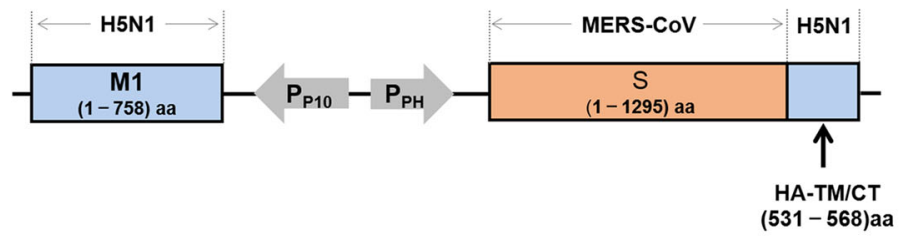

C

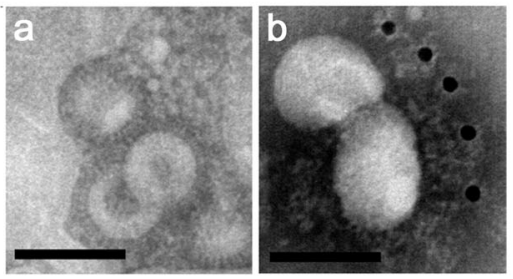

D

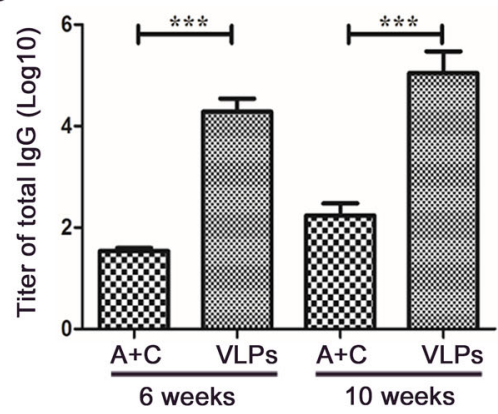

B

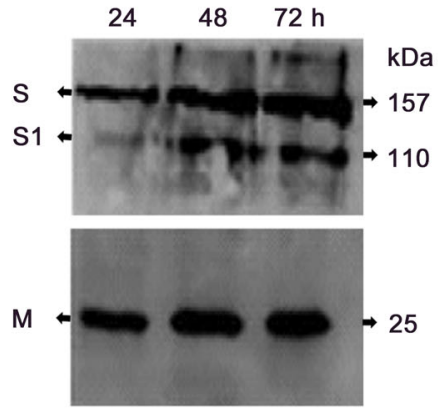

E

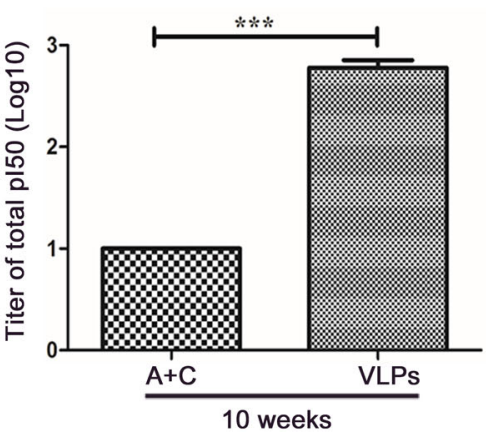

Fig. 1 Robust humoral immunity in mice induced by the novel chimeric virus-like particles (cVLPs) vaccine candidate for Middle East respiratory syndrome coronavirus (MERS-CoV) infection. A A schematic representation of the recombinant baculoviral expression system expressing Middle East respiratory syndrome coronaviral $\mathrm{S}$ and avian influenza matrix 1 based on the recombinant plasmid pFastBacDual. B Expression of the recombinant protein in infected cells at an optimal MOI (MOI $=5$ ) analyzed by Western blot. Proteins were harvested at different time points (24, 72 and $96 \mathrm{~h})$. S protein expression was analyzed by immunoblotting using a monoclonal anti-S (MERS-CoV) and anti-M1 (H5N1) antibodies. The top panel indicate $\mathrm{S}$ expression; bottom panel, M1 expression. C The morphology of cVLPs assessed via transmission electron microscopy and immuno-electron microscopy. Figure $1 \mathbf{C}$-a shows the results of negative staining assessed via electron microscopy. Figure $1 \mathbf{C}-\mathrm{b}$ shows the results of immuno-electron microscopy. cVLPs were incubated with murine monoclonal antibodies against MERS-CoV S protein and probed using a gold-labeled goat anti-mouse IgG antibody. Bar $=100 \mathrm{~nm}$. D S-specific IgG antibodies detected by ELISA in the serum of immunized mice. The titers of S-specific total IgG in the serum of mice 2 weeks after the second (6 weeks) and the third immunization (10 weeks). E Neutralizing antibody titer in the serum of mice, detected by a pseudovirus neutralization assay of MERS-CoV 2 weeks after the third immunization (10 weeks), indicated as pI50. A $+\mathrm{C}$ implies the control group of mice injected with adjuvants aluminum (A) plus $\mathrm{CpG}(\mathrm{C})$. 
were observed on the $\mathrm{cVLP}_{\mathrm{S}}$ (Fig. 1C-b). These results indicate that chimeric MERS VLPs are morphologically similar to native MERS-CoV (https://www.cdc.gov/cor onavirus/mers/photos.html).

To assess the immunogenicity of $\mathrm{cVLP}_{\mathrm{S}}$ in mice, 6-8week-old female $\mathrm{BALB} / \mathrm{c}$ mice were intramuscularly injected with $1 \mu \mathrm{g}$ of cVLPs of MERS-S adjuvanted with $100 \mu \mathrm{L}$ of aluminum (A) and $10 \mu \mathrm{g}$ of $\mathrm{CpG}$ (C). Simultaneously, the mice of control group inoculated with adjutants $(\mathrm{A}+\mathrm{C})$ only. All mice were immunized thrice at 4 -week intervals. Mice were bled via the venae angularis and serum was harvested from whole blood to determine IgG levels via enzyme-linked immunosorbent assay (ELISA) and neutralization activity was assessed via the pseudovirus neutralization assay for MERS-CoV (Lan et al. 2014; Deng et al. 2018) As shown in Fig. 1D, 2 weeks after the second immunization, the total S proteinspecific IgG titer approached 1:80,000 in the VLP group, being significantly higher than that in the control group. After the third immunization, the IgG titer was further elevated (more than $10^{5}$ ). More importantly, high titers of neutralization antibodies (at a serum dilution of $1: 320,>50 \%$ neutralizing activity based on the pseudovirus neutralization assay, indicated as $\mathrm{pI}_{50}=320$ ) were also detected in mice immunized with cVLPs (Fig. 1E).

We developed immunogenic cVLPs of MERS-S via coexpression of H5N1 M1 protein and MERS-CoV S protein in a baculoviral expression system. The present results indicate that cVLPs with surface expression of MERS-CoV $\mathrm{S}$ protein, morphologically emulating the native virus, are promising candidates for prophylactic vaccines for MERS$\mathrm{CoV}$ infections. A similar strategy was adopted to formulate cVLPs of SARS-CoV (Liu et al. 2011). The present results thus indicate that cVLPs of MERS-S mimic wild type viral morphology and induce robust $\mathrm{S}$-specific humoral immune responses in mice. In the future study, we intend to use a similar platform to develop cVLPs of other $\mathrm{CoVs}$ or highly pathogenic viruses to further validate the present strategy and to purify cVLPs of MERS-S on a large scale to evaluate the protective effect of cVLPs in animal models of infection.
Acknowledgements This work was supported by the Megaproject for Infectious Disease Research of China (2016ZX10004001-003 and 2014ZX10004001-002 to W.T.), and the National Key Research and Development Program of China (2016YFD0500300 to W.T., 2016YFC1200901 to Y.D., and 2016YFC1200200 to B.H). The funders had no role in study design, data collection and analysis, decision to publish, or preparation of the manuscript.

\section{Compliance with Ethics Standards}

Conflict of interest The authors declare that they have no conflict of interest.

Animal and Human Rights Statement Animal studies were conducted in strict accordance with the Guide for the Care and Use of Laboratory Animals of the People's Republic of China. The study protocol was approved by the Committee on the Ethics of Animal Experiments of the Chinese Centre for Disease Control and Prevention.

\section{References}

Deng Y, Lan J, Bao L, Huang B, Ye F, Chen Y, Yao Y, Wang W, Qin C, Tan W (2018) Enhanced protection in mice induced by immunization with inactivated whole viruses compare to spike protein of middle east respiratory syndrome coronavirus. Emerg Microbes Infect 7:60

Lan J, Deng Y, Chen H, Lu G, Wang W, Guo X, Lu Z, Gao GF, Tan W (2014) Tailoring subunit vaccine immunity with adjuvant combinations and delivery routes using the Middle East respiratory coronavirus (MERS-CoV) receptor-binding domain as an antigen. PLoS ONE 9:e112602

Liu YV, Massare MJ, Barnard DL, Kort T, Nathan M, Wang L, Smith G (2011) Chimeric severe acute respiratory syndrome coronavirus (SARS-CoV) $\mathrm{S}$ glycoprotein and influenza matrix 1 efficiently form virus-like particles (VLPs) that protect mice against challenge with SARS-CoV. Vaccine 29:6606-6613

Okba NM, Raj VS, Haagmans BL (2017) Middle East respiratory syndrome coronavirus vaccines: current status and novel approaches. Curr Opin Virol 23:49-58

van Boheemen S, de Graaf M, Lauber C, Bestebroer TM, Raj VS, Zaki AM, Osterhaus AD, Haagmans BL, Gorbalenya AE, Snijder EJ, Fouchier RA (2012) Genomic characterization of a newly discovered coronavirus associated with acute respiratory distress syndrome in humans. MBio 3:e00473-12

Wang C, Zhang X, Gai W, Zhao Y, Wang H, Wang H, Feng N, Chi H, Qiu B, Li N, Wang T, Gao Y, Yang S, Xia X (2017) MERS$\mathrm{CoV}$ virus-like particles produced in insect cells induce specific humoural and cellular imminity in rhesus macaques. Oncotarget 8:12686-12694 